\title{
Las aulas virtuales en la docencia de una universidad presencial: la visión del alumnado
}

\section{Virtual classrooms in face-to-face tertiary education: the student perspective}

\author{
Manuel Area Moreira \\ María Belén San Nicolás Santos \\ Ana Luisa Sanabria Mesa \\ Universidad de La Laguna (España)
}

\section{Resumen}

Este artículo aborda el análisis pedagógico de los entornos, espacios o aulas virtuales en la docencia presencial universitaria. Es continuación de otros trabajos previos de los autores que, desde hace casi una década, revisan el proceso de integración y generalización de las aulas virtuales en una universidad que oferta la mayoría de sus titulaciones en la modalidad de enseñanza presencial. En este estudio se han seleccionado 12 casos de aulas virtuales de distintos campos o ramas de conocimiento analizadas con un instrumento de observación diseñado ad hoc y se han realizado doce entrevistas grupales al alumnado matriculado en las mismas. De los datos obtenidos se infiere que estos entornos o aulas virtuales responden a un modelo pedagógico de enseñanza expositiva caracterizado por ser un repositorio de objetos digitales que empaquetan el conocimiento, por ser el espacio a través del que el alumnado entrega las tareas o trabajos que les demandan, y por existir un bajo nivel de interacción social entre los docentes y los estudiantes durante todo el proceso. Se concluye con recomendaciones para el desarrollo de políticas institucionales en universidades presenciales destinadas a la generalización entre su profesorado de modelos pedagógicos innovadores en estos entornos formativos digitales.

Palabras clave: aula virtual; docencia universitaria; e-learning; enseñanza semipresencial; educación digital.

\begin{abstract}
This article presents the pedagogical analysis of environments, spaces or virtual classrooms. It is a continuation of other previous works by the authors who, for almost a decade, have been checking the process of integration and generalization of the virtual classrooms by the entire faculty of a university offering all of their degrees in face-to-face teaching. In this study, 12 cases of virtual classrooms from different fields or branches of knowledge that were analyzed with a specific observation instrument were selected and twelve group interviews were conducted with the students enrolled in them. It has been found that these environments
\end{abstract}


or virtual classrooms respond to a pedagogical model of expository teaching characterized by being a repository of digital objects that pack knowledge, as it is the resource through which students deliver the tasks or jobs that they demand, and with a low level of social interaction between teachers and students. It concludes with recommendations for the development of institutional policies that stimulate the development of innovative pedagogical models of teaching through technologies.

Keywords: virtual classroom; higher education; elearning; blended learning; digital education.

En las universidades presenciales, entre las múltiples formas, modalidades y estrategias de uso pedagógico de las tecnologías digitales (MOOC, flipped classroom, realidad aumentada, PLE, microcursos online, entornos adaptativos, analíticas del aprendizaje,...), la docencia presencial apoyada en un aula virtual dentro de un campus digital institucional es la experiencia y práctica más generalizada entre el profesorado y el alumnado en las titulaciones oficiales tanto de grado como postgrado. La Conferencia de Rectores de Universidades Españolas, en el informe UNIVERSITIC 2015, señalaba que desde el año 2013 más del 90\% del profesorado de las universidades utiliza la plataforma de docencia virtual institucional, lo que constituye una importante consolidación y generalización de esta modalidad educativa (Piriz, 2015).

En este artículo realizamos un análisis de una muestra de entornos, espacios o aulas virtuales de asignaturas en el contexto de enseñanza universitaria presencial. Es continuación de otros trabajos previos de los autores que, desde hace casi una década, revisan el proceso de integración y generalización de las aulas virtuales por el conjunto del profesorado de una universidad que oferta la gran parte de sus titulaciones en la modalidad de educación presencial. El estudio presentado se basa en una selección de doce casos de aulas virtuales combinando su análisis con la visión que tienen los estudiantes sobre su uso en la docencia universitaria.

\section{Las aulas virtuales como escenarios para la docencia universitaria}

Un entorno, espacio o aula virtual de formación se refiere a un lugar acotado y reconocible en el ciberespacio que posee una identidad y estructura definida con fines educativos. Es un entorno virtual creado con la intencionalidad pedagógica de estimular, guiar o supervisar un proceso de aprendizaje de modo formalizado (Area y Adell, 2009). En el contexto español, contamos con una destacable fundamentación de las bases pedagógicas de la enseñanza o educación virtual, destacando una serie de textos como: Duart y Sangrá (2000); Aguaded y Cabero (2002; 2013); Salinas (2004); Cabero (2006); García Aretio (2007); Barberá (2008); Coll y Monereo (2008); Revuelta y Pérez (2009); Suárez y Gros (2013); Salinas, Darder y Benito (2015), por citar algunos de los más difundidos y citados. 
La literatura pedagógica anteriormente citada nos indica que un entorno, aula o espacio de enseñanza-aprendizaje en línea se compone básicamente por cuatro elementos o dimensiones: las actividades o tareas, los materiales didácticos u objetos de aprendizaje digitales, los procesos comunicativos y la tutorización evaluativa:

- Los entornos educativos online o virtuales debieran facilitar procesos de aprendizaje basados en la actividad y construcción del conocimiento (Gros, 2002; Mauri y Onrubia, 2008). Esto implicará que dichos entornos se organicen y demanden a los estudiantes la cumplimentación de tareas o actividades de diversa naturaleza, más que la mera recepción de contenidos o informaciones (Cabero y Román, 2006).

- Los entornos de docencia virtuales deben incorporar materiales didácticos caracterizados por presentar el conocimiento mediantes lenguajes y formatos variados como son los textos, las imágenes, los audiovisuales, las animaciones (Cabero y Gisbert, 2005).

- Los entornos de aprendizaje virtuales deben ser escenarios de comunicación e interacción social ricos, variados y en permanente interacción entre los estudiantes y el docente (Revuelta y Pérez, 2009; Suárez y Gros, 2013).

- La función docente principal, además del diseño del entorno, consiste en animar, tutorizar y evaluar las actividades de participación social y producción intelectual de los estudiantes (Adell y Sales, 1999). En otras palabras, saber gestionar la interacción social del grupo clase y de cada uno de los estudiantes, evaluándolos de forma continuada en su experiencia de aprendizaje en dicho entorno o aula virtual Gisbert (2002).

\section{Las políticas en las universidades presenciales como condicionante}

Además de los anteriores factores que inciden en la calidad educativa de los entornos formativos en línea, también sabemos que el contexto institucional bajo el cual se crean y utilizan estas aulas virtuales es altamente relevante, al menos en las universidades de docencia presencial. Las políticas universitarias con relación a estimular, facilitar y generalizar la formación del profesorado, el impulso a titulaciones a distancia y semipresenciales, la creación de condiciones organizativas y el reconocimiento de la docencia digital son otro importante factor a tener en cuenta (Bates y Sangrá, 2011).

En esta dirección, Pallof y Pratt (2013) establecen una serie de lecciones o conclusiones derivadas de más de una década de experiencias, donde señalan que la educación en línea va a coexistir con la enseñanza presencial y que es muy relevante una política institucional de apoyo a la enseñanza virtual en el contexto universitario.

De modo similar, el informe destinado a la Comisión Europea elaborado por un grupo de expertos sobre la calidad en educación superior (Comisión Europea, 2014), recomienda que: 
- La integración de las tecnologías digitales y las pedagogías debería ser un elemento integral de las estrategias de las instituciones educativas para la enseñanza y el aprendizaje. Los objetivos y metas deben ser claros y contar con estructuras de apoyo organizativas para impulsar la implementación.

- Todo el personal docente en instituciones de educación superior debe recibir capacitación en tecnologías y pedagogías como parte de la formación inicial y el desarrollo profesional continuo.

- Los gobiernos y las instituciones de educación superior deben trabajar hacia el acceso abierto y completo de recursos educativos.

\section{El contexto institucional del estudio: El caso de una universidad presencial}

El estudio empírico presentado se desarrolla en el contexto de la Universidad de La Laguna (ULL). Se trata de una universidad con más de dos siglos de antigüedad, eminentemente presencial. Desde el año 2005 la universidad cuenta con un Campus Virtual (basado en sistema de gestión de curso Moodle) para todas las titulaciones, donde los profesores pueden habilitar los correspondientes espacios de sus asignaturas y dar acceso al alumnado para su uso como recursos de apoyo a la docencia.

La primera versión del Campus Virtual de la ULL se creó en el año 2001 implementada por el Laboratorio de Educación y Nuevas Tecnologías de la Universidad de la Laguna (Edullab). En dicho Campus Virtual se tenía acceso, entre otros, a una serie de cursos de doctorado en línea, diseñados para la plataforma WebCT, así como a distintos sitios web de asignaturas concretas, desarrolladas por el profesorado de la ULL en formato HTML y clasificadas por campos científicos.

En el año 2005, la Junta de Gobierno de la ULL aprobó la creación de un Campus Virtual institucional gestionado por la UDV (Unidad para la Docencia Virtual), que realizó la primera convocatoria de proyectos de docencia virtual destinados a asignaturas de cualquier titulación oficial de la ULL a través del Vicerrectorado de Planes de Estudios y Títulos Propios. En ese primer año, a modo experimental, se crearon 45 aulas virtuales. En el curso siguiente se repitió la convocatoria de proyectos piloto generándose un total de 141 aulas.

En el curso 2016-2017 existían un total de 2.686 aulas virtuales activas en uso en las titulaciones ofertadas. Este dato atestigua una importante implantación de las aulas virtuales en la docencia presencial, ya que esta universidad cuenta con una población de 1.800 docentes. La Unidad para la Docencia Virtual es el servicio institucional de referencia para la docencia en línea o virtual en la ULL. Aunque la mayor parte de las titulaciones de la ULL se desarrollan en una modalidad presencial, el Campus Virtual juega un papel muy importante como recurso de apoyo a la docencia. Asimismo, en los últimos años han surgido algunas titulaciones 
oficiales de postgrado que se desarrollan en línea tanto en la modalidad de educación a distancia como de enseñanza semipresencial o de b-learning.

\section{Diseño y metodología}

Este estudio fue diseñado con la finalidad de analizar las dimensiones pedagógicas de una muestra de aulas virtuales de apoyo a la docencia presencial así como de las perspectivas del alumnado sobre las mismas. Se trata de un estudio cualitativo de casos donde se analizaron las aulas virtuales a través de un instrumento construido ad hoc (véase el anexo 1), a modo de observación sistemática de los recursos y el desarrollo de las prácticas en las aulas virtuales (Gorad y Taylor, 2004). Este estudio fue complementado con la realización de entrevistas grupales a una muestra del alumnado que cursaba sus asignaturas a través de dichas aulas virtuales con la intención de identificar sus visiones con relación a la utilidad de dichos entornos en el proceso de aprendizaje.

\section{Antecedentes}

Los autores del artículo tienen una amplia experiencia previa en analizar y explorar las aulas virtuales creadas en la ULL. A solicitud de la UDV, en el periodo 2005-2007 realizamos la primera evaluación del Campus Virtual de la ULL en el que se analizaron un total de 45 aulas virtuales en el curso 2005-2006 y 71 en el curso 2006-2007. En el mismo, se analizaron las características de las aulas virtuales de las asignaturas en función de las Ramas de Conocimiento a las que pertenecía. En esta misma línea se ha realizado otros análisis con posterioridad que pueden consultarse (Area, San Nicolás y Fariña 2008; 2010; Area, Sanabria y González, 2008; San Nicolás, Fariña y González, 2011; Fariña, González y Area, 2013).

\section{Objetivo y cuestiones del estudio}

El objetivo central fue realizar una aproximación diagnóstica o caracterizadora del tipo de entornos virtuales que se ofrece al alumnado en distintas titulaciones de grado presenciales en la ULL, donde nos planteamos las siguientes cuestiones o preguntas de investigación:

a. ¿qué elementos, recursos y dimensiones caracterizan a las aulas virtuales de la docencia presencial?

b. ¿existen rasgos diferenciadores entre aulas virtuales en función de la rama o campo de conocimiento?

c. ¿̇cuál es el modelo pedagógico de enseñanza-aprendizaje que subyace en las aulas virtuales ofertadas en la docencia presencial? 
d. ¿́cuál es la perspectiva del alumnado sobre el potencial y valor de las aulas virtuales en su aprendizaje?

\section{La selección de la muestra}

El criterio inicial de selección de la muestra fue buscar profesorado que hubiera participado en varias convocatorias de los Proyectos de Docencia Virtual (PRODOVI) convocados por la UDV. En este proceso surgieron algunas dificultades, ya que algunos profesores no estaban dispuestos a dar acceso a sus aulas virtuales y tampoco a permitir el acceso al alumnado para el desarrollo de las entrevistas grupales. Finalmente, se consiguió el permiso y colaboración del profesorado de 12 asignaturas de distintas titulaciones de Grado en la ULL, de modo que pudimos acceder a las aulas virtuales y a los estudiantes inscritos en las mismas. Los datos concretos de cada uno de estos casos pueden verse en la tabla 1.

Tabla 1. Distribución y características de casos por rama de conocimiento

\begin{tabular}{|c|c|c|c|c|c|c|}
\hline $\begin{array}{c}\text { Rama de } \\
\text { conocimiento }\end{array}$ & $\begin{array}{l}\text { Titulación } \\
\text { de las aulas } \\
\text { virtuales } \\
\text { analizadas }\end{array}$ & Curso & $\begin{array}{c}\mathrm{N}^{\mathrm{O}} \\
\text { Profesores } \\
\text { en aula } \\
\text { virtual }\end{array}$ & $\begin{array}{c}\mathrm{N}^{\mathrm{O}} \\
\text { estudiantes } \\
\text { matriculados } \\
\text { en aula } \\
\text { virtual }\end{array}$ & $\begin{array}{c}\mathrm{N}^{\mathrm{O}} \\
\text { estudiantes } \\
\text { en entrevista } \\
\text { grupal }\end{array}$ & $\begin{array}{l}\text { Duración } \\
\text { entrevistas }\end{array}$ \\
\hline \multirow{3}{*}{$\begin{array}{l}\text { Artes y } \\
\text { Humanidades }\end{array}$} & $\begin{array}{l}\text { Licenciatura } \\
\text { en Historia }\end{array}$ & Optativa & 1 & 25 & 11 & $28: 29$ \\
\hline & $\begin{array}{l}\text { Grado en } \\
\text { Historia } \\
\end{array}$ & $1^{0}$ & 2 & 70 & 8 & $41: 12$ \\
\hline & $\begin{array}{l}\text { Grado en } \\
\text { Español: } \\
\text { Lengua y } \\
\text { Literatura } \\
\end{array}$ & Optativa & 1 & 25 & 10 & $54: 25$ \\
\hline Ciencias & $\begin{array}{l}\text { Licenciatura } \\
\text { en Química }\end{array}$ & $4^{o}$ & 4 & 20 & 9 & 31:07 \\
\hline $\begin{array}{l}\text { Ciencias de la } \\
\text { Salud }\end{array}$ & $\begin{array}{l}\text { Grado en } \\
\text { Medicina }\end{array}$ & $1^{0}$ & 5 & 170 & 12 & $46: 10$ \\
\hline $\begin{array}{l}\text { Licenciatura } \\
\text { en Farmacia }\end{array}$ & Optativa & 1 & 13 & 11 & $27: 32$ & \\
\hline $\begin{array}{l}\text { Licenciatura } \\
\text { en Psicología }\end{array}$ & $4^{0}$ & 4 & 217 & 5 & $45: 42$ & \\
\hline $\begin{array}{l}\text { Ingeniería y } \\
\text { Arquitectura }\end{array}$ & $\begin{array}{l}\text { Ingeniería } \\
\text { Técnica } \\
\text { Industrial } \\
\text { (Especialidad } \\
\text { Mecánica) }\end{array}$ & $3^{o}$ & 2 & 96 & 7 & $50: 45$ \\
\hline
\end{tabular}




\begin{tabular}{|c|c|c|c|c|c|c|}
\hline $\begin{array}{c}\text { Rama de } \\
\text { conocimiento }\end{array}$ & $\begin{array}{l}\text { Titulación } \\
\text { de las aulas } \\
\text { virtuales } \\
\text { analizadas }\end{array}$ & Curso & $\begin{array}{c}\mathrm{N}^{\mathrm{o}} \\
\text { Profesores } \\
\text { en aula } \\
\text { virtual }\end{array}$ & $\begin{array}{c}\mathrm{N}^{\mathrm{O}} \\
\text { estudiantes } \\
\text { matriculados } \\
\text { en aula } \\
\text { virtual }\end{array}$ & \begin{tabular}{|c|}
$\mathrm{N}^{\mathrm{O}}$ \\
estudiantes \\
en entrevista \\
grupal
\end{tabular} & $\begin{array}{l}\text { Duración } \\
\text { entrevistas }\end{array}$ \\
\hline $\begin{array}{l}\text { Grado de } \\
\text { Ingeniería } \\
\text { Informática }\end{array}$ & $1^{0}$ & 3 & 79 & 13 & 28:21 & \\
\hline $\begin{array}{l}\text { Ciencias } \\
\text { Sociales y } \\
\text { Jurídicas }\end{array}$ & $\begin{array}{l}\text { Grado de } \\
\text { Maestro en } \\
\text { Educación } \\
\text { Primaria } \\
\end{array}$ & $1^{0}$ & 3 & 97 & 10 & $36: 07$ \\
\hline $\begin{array}{l}\text { Asignatura } \\
\text { de Libre } \\
\text { configuración } \\
\text { de la Facultad } \\
\text { de Educación }\end{array}$ & Optativa & 1 & 26 & 11 & 31:01 & \\
\hline $\begin{array}{l}\text { Grado en } \\
\text { Derecho }\end{array}$ & $1^{\mathrm{o}}$ & 1 & 127 & 12 & $43: 17$ & \\
\hline
\end{tabular}

\section{Instrumentos de recogida y análisis de datos}

La recogida de datos para el análisis de las aulas virtuales se realizó a través de un instrumento de observación, específicamente creado por los autores para este tipo de aulas virtuales, que puede verse en el Anexo 1. El proceso de construcción y validación de este instrumento fue realizado a través de estudios previos desarrollados en años anteriores donde se exploraron diferentes muestras de aulas virtuales del Campus Virtual de la ULL en los que se fue contrastando y afinando el instrumento (Area, San Nicolás y Fariña 2008; 2010; Fariña, González, y Area, 2013). Las dimensiones contempladas en el mismo son las siguientes: datos de identificación, objetos digitales y recursos de información, organización didáctica, modelo de aprendizaje, tareas y actividades, recursos de comunicación, recursos externos, funciones de los participantes, evaluación y tutorización, los aspectos gráficos, así como el modelo pedagógico.

Para el segundo estudio, se realizaron 12 entrevistas grupales (Aigneren, 2009) al alumnado de cada una de las aulas virtuales analizadas, para recabar la información relativa a las opiniones del alumnado sobre las aulas virtuales como recurso de apoyo al aprendizaje. Las dimensiones y preguntas formuladas en cada entrevista grupal pueden verse en el Anexo 2. Las entrevistas grupales se realizaron a grupos configurados entre 6-11 alumnos y la duración de cada una de las mismas osciló entre los 30-60 minutos. Estas fueron grabadas en formato audio para su posterior transcripción escrita, y análisis a través de la aplicación Atlas.ti. 


\section{Resultados}

Los resultados los hemos sintetizado y están presentados en función de los cuatro objetivos/preguntas de investigación previamente señalados:

\section{a. ¿Cuáles son los elementos, recursos y dimensiones destacables de las aulas virtuales universitarias de la enseñanza presencial?}

\begin{tabular}{|c|c|}
\hline Dimensiones & Caracteristicas de las aulas virtuales \\
\hline $\begin{array}{l}\text { Los objetos } \\
\text { y recursos } \\
\text { digitales de } \\
\text { información }\end{array}$ & $\begin{array}{l}\text { Los objetos digitales más utilizados en las aulas virtuales son los } \\
\text { documentos de texto y las presentaciones multimedia de diapositivas. Los } \\
\text { de naturaleza audiovisual son minoritarios. Ello manifiesta que la forma } \\
\text { de presentación del conocimiento sigue siendo predominantemente } \\
\text { textual lo que supone que el material didáctico virtual imita o } \\
\text { reproduce los objetos o artefactos impresos tradicionales de la docencia } \\
\text { universitaria (apuntes y libros). }\end{array}$ \\
\hline $\begin{array}{l}\text { Agrupamiento } \\
\text { del alumnado }\end{array}$ & $\begin{array}{l}\text { En todas las aulas virtuales se utiliza un agrupamiento individual para } \\
\text { las actividades. Solo en casos puntuales se utiliza un agrupamiento en } \\
\text { pequeño grupo fijo, es decir, se configuran pequeños grupos de trabajo } \\
\text { que no cambian cuando se propone una actividad nueva, sino que se } \\
\text { mantiene a lo largo de toda la asignatura. Todo ello pone de manifiesto } \\
\text { que estas aulas siguen manteniendo una concepción del aprendizaje } \\
\text { basada en la acción individual de cada estudiante sobre el conocimiento } \\
\text { desconsiderando la potencialidad del aprendizaje colaborativo y grupal. }\end{array}$ \\
\hline $\begin{array}{l}\text { Actividades } \\
\text { y tareas } \\
\text { demandas }\end{array}$ & $\begin{array}{l}\text { Las actividades realizadas a través del aula virtual consisten en la subida } \\
\text { de archivos. Las demandas en estas tareas difieren de una titulación a } \\
\text { otras, pero es común la entrega de informes de prácticas. } \\
\text { En la mayor parte de los casos el profesorado solicita el desarrollo de } \\
\text { ensayo o informes sobre aspectos trabajados en la asignatura, la mayor } \\
\text { parte de estos están relacionados con prácticas. En la mitad de los casos, } \\
\text { la actividad más común sería los cuestionarios y la de resolución de } \\
\text { ejercicios. }\end{array}$ \\
\hline $\begin{array}{l}\text { Recursos de } \\
\text { comunicación }\end{array}$ & $\begin{array}{l}\text { Los recursos de comunicación son en general muy poco utilizados para la } \\
\text { interacción entre el alumnado y el profesorado o solo entre el alumnado. } \\
\text { En todas las aulas virtuales se incorpora el foro de novedades a través } \\
\text { del que el profesorado envía las comunicaciones al alumnado. Este foro } \\
\text { es unidireccional, por lo que los estudiantes no pueden intervenir en el } \\
\text { mismo. } \\
\text { En ninguno de los casos se utilizan recursos de comunicación síncrona } \\
\text { como la videoconferencia o el chat. }\end{array}$ \\
\hline
\end{tabular}




\begin{tabular}{|c|c|}
\hline Dimensiones & Características de las aulas virtuales \\
\hline $\begin{array}{l}\text { Organización } \\
\text { didáctica }\end{array}$ & $\begin{array}{l}\text { La estructura utilizada en la mayoría de las aulas virtuales es la temática, } \\
\text { donde la secuencia de temas es el criterio de organización; en algunos } \\
\text { casos se organiza también por tareas, identificando las principales } \\
\text { actividades a las que se dedica el contenido de cada bloque; en otros, no } \\
\text { hay una estructura clara, donde se diferencie el contenido que contiene } \\
\text { cada uno de los bloques. }\end{array}$ \\
\hline $\begin{array}{l}\text { Funciones } \\
\text { de los } \\
\text { participantes }\end{array}$ & $\begin{array}{l}\text { Funciones del docente: en la mayoría de las aulas virtuales analizadas, } \\
\text { el papel principal del profesorado es el de transmisor de conocimientos, } \\
\text { sin embargo, este no se da de forma exclusiva, sino que coexisten con el } \\
\text { desarrollo de funciones como guía del aprendizaje en las actividades o } \\
\text { como curadores de contenido de relevancia para la asignatura. } \\
\text { Funciones del alumnado: el papel del alumnado está mediado por la } \\
\text { metodología y las actividades planteadas. Desde este punto de vista, } \\
\text { el papel del alumnado en el aula virtual consiste principalmente en } \\
\text { desarrollar las actividades planteadas y leer los textos y materiales } \\
\text { facilitados por el profesorado. }\end{array}$ \\
\hline $\begin{array}{l}\text { Evaluación, } \\
\text { seguimiento y } \\
\text { tutorización: }\end{array}$ & $\begin{array}{l}\text { Solo en la mitad de las aulas virtuales se recogen evidencias de la } \\
\text { evaluación, seguimiento y tutorización del aprendizaje del alumnado. } \\
\text { La evaluación del alumnado se realiza principalmente a través de la } \\
\text { entrega de trabajos o tareas y de un examen final tipo test en forma de } \\
\text { cuestionario de preguntas de opción múltiple, que el profesorado utiliza } \\
\text { para comprobar la adquisición de conceptos o resolución de problemas. } \\
\text { El profesorado no suele facilitar las calificaciones a través del libro de } \\
\text { calificaciones, en algunos casos se hace colgando un archivo con las notas } \\
\text { en el aula virtual. Apenas hay feedback o tutorización continuada. }\end{array}$ \\
\hline $\begin{array}{l}\text { Aspectos } \\
\text { gráficos }\end{array}$ & $\begin{array}{l}\text { La interface en todas las aulas es predominantemente textual. En general } \\
\text { no se utilizan imágenes, pero sí se destacan títulos distinguiendo tamaño } \\
\text { y color de letra. Solo en algunos casos de utilizan epígrafes que ayuden en } \\
\text { la organización del contenido presentado en los temas. }\end{array}$ \\
\hline $\begin{array}{l}\text { Recursos } \\
\text { externos al aula } \\
\text { virtual }\end{array}$ & $\begin{array}{l}\text { Los recursos más utilizados son los textos, las presentaciones y los } \\
\text { enlaces a recursos externos al aula virtual. No se han recogido materiales } \\
\text { interactivos o audiovisuales. }\end{array}$ \\
\hline
\end{tabular}

\section{b. ¿Existen diferencias entre aulas virtuales en función del área o rama de conocimiento?}

No hemos encontrado que existan diferencias singulares de las características de las aulas virtuales en función de las distintas ramas de conocimiento. En otras palabras, no existen modelos didácticos, organizativos o comunicacionales compartidos o comunes entre las aulas de cada una de las ramas de conocimiento que los convierta en diferenciales respecto a los otros campos. En todo caso, hemos encontrado algunos matices o rasgos en alguna variable concreta como es el tipo de tarea o actividad solicitada. Así por ejemplo, en Ciencias Sociales o en Artes y 
Humanidades se demanda la realización de ensayos; y en Ciencias, Ciencias de la Salud e Ingeniería y Arquitectura, se solicita la realización de informes generales o informes de prácticas y resolución de problemas.

Las prácticas que los estudiantes realizan en las aulas virtuales no varían de unas titulaciones a otras. En este sentido, se constata que las actividades y recursos son similares y también las dificultades con las que se encuentran. Atendiendo a las diferencias existentes cabe decir que en general el alumnado, en especial en la rama de Ciencias, pone en valor el contacto cara a cara con el profesorado para resolver las dudas relacionadas con la resolución de ejercicios, mientras que les resulta más difícil plantear dichas dudas o problemas a través de un foro en el aula virtual.

En la rama de conocimiento de Artes y Humanidades, el alumnado utiliza en mayor medida un programa de edición de imágenes, algo que puede estar relacionado con los contenidos específicos de la titulación o con los intereses de los estudiantes. Mientras que en la rama de conocimiento de Ingeniería y Arquitectura, el alumnado dedica aproximadamente el doble de tiempo al uso del aula virtual que el alumnado del resto de ramas de conocimiento.

\section{c. ¿Cuál es el modelo pedagógico de enseñanza-aprendizaje que subyace en las aulas virtuales ofertadas en la docencia presencial?}

El modelo pedagógico desarrollado en la mayor parte de las aulas analizadas pudiéramos señalar que responde a una concepción expositiva del conocimiento y de aprendizaje por recepción. Evidentemente, hay matices diferenciales de este modelo ya que en algunas de las mismas se mezclan estrategias de distinto tipo: por un lado, un modelo de aprendizaje por recepción asociado a la presencia de numerosos documentos de texto y presentaciones que el alumnado debe utilizar para su estudio; y por otro lado, la demanda de tareas o actividades que el alumnado debe realizar a lo largo de la asignatura y que generalmente forman parte de la evaluación continua de la misma.

La principal funcionalidad o utilidad didáctica de las aulas virtuales es albergar los materiales didácticos de estudio de la asignatura y facilitar un espacio para recopilar las tareas que el alumnado debe desarrollar. Los procesos comunicativos solo se realizan en pocas aulas virtuales y de forma esporádica, pero no es el objetivo principal. La estructura utilizada en las aulas virtuales es temática, siguiendo en general una secuencia que responde a la de los temas de la asignatura. Sin embargo, también hay casos que, aunque utilizan esta estructura, dedica cada uno de los bloques a diferentes tipos de actividades relacionadas con la temática, organizando, por ejemplo, los contenidos teóricos principales en un bloque diferenciado de los materiales complementarios.

Los recursos de comunicación son muy poco usados por el alumnado, pero también es cierto que el profesorado no suele habilitar espacios destinados a la comunicación donde los estudiantes tengan posibilidad de intervenir para plantear 
dudas o desarrollar, por ejemplo, actividades de debate. Las actividades más comunes en las aulas virtuales son las tareas individuales. Las actividades colaborativas que se pueden configurar en las aulas virtuales como wikis, talleres, glosarios o bases de datos, son en general poco usadas.

\section{d. La visión del alumnado sobre las aulas virtuales}

\begin{tabular}{|c|c|}
\hline Dimensiones & Visiones del alumnado \\
\hline $\begin{array}{l}\text { Uso personal } \\
\text { de las TIC }\end{array}$ & $\begin{array}{l}\text { La mayoría del alumnado dispone de su propio ordenador y conexión a } \\
\text { Internet en el hogar. El uso que realizan de las TIC se focaliza en las redes } \\
\text { sociales, consulta del correo electrónico, prensa en línea y búsquedas. En } \\
\text { menor medida participan en blogs, solo en casos puntuales como en la } \\
\text { Licenciatura de Psicología o el Grado en Derecho. }\end{array}$ \\
\hline $\begin{array}{l}\text { Experiencia } \\
\text { previa con } \\
\text { aulas virtuales }\end{array}$ & $\begin{array}{l}\text { El grado de experiencia previa del alumnado es variable, para una parte } \\
\text { importante del alumnado es su primera vez usando aulas virtuales, sin } \\
\text { embargo otros estudiantes han tenido experiencias previas de utilización } \\
\text { de las aulas virtuales en secundaria, por lo que conocen la dinámica y el } \\
\text { funcionamiento. }\end{array}$ \\
\hline $\begin{array}{l}\text { Características } \\
\text { y usos del aula } \\
\text { virtual }\end{array}$ & $\begin{array}{l}\text { Destaca el uso de las aulas virtuales para facilitar el acceso a los materiales } \\
\text { de estudio de la asignatura. } \\
\text { Contenidos y recursos de información: el alumnado valora positivamente } \\
\text { la disponibilidad de presentaciones, textos y apuntes, en muchos casos } \\
\text { las aulas virtuales se han convertido en un repositorio de este tipo de } \\
\text { información. } \\
\text { Actividades: el alumnado considera que no se aprovechan } \\
\text { suficientemente las posibilidades de las aulas virtuales para integrar } \\
\text { recursos disponibles en línea, enlaces externos al aula virtual, elementos } \\
\text { multimedia o audiovisuales, así como las posibilidades de interacción } \\
\text { entre el alumnado y entre estos y el profesorado a través de foro o de } \\
\text { recursos de trabajo colaborativo en línea, etc. } \\
\text { Recursos de comunicación: los recursos de comunicación de las aulas } \\
\text { virtuales son muy poco utilizados. En general se valora el contacto cara a } \\
\text { cara y es posible que estos procesos se trasladen al contexto del aula. } \\
\text { Organización didáctica: es común entre el alumnado la queja de la } \\
\text { sobrecarga de información y la mala organización del contenido que } \\
\text { impide identificar qué elementos forman parte de los materiales de } \\
\text { estudio y lo que son recursos complementarios, lo que genera confusión } \\
\text { entre los estudiantes. } \\
\text { Evaluación, seguimiento y tutorización: Las funcionalidades de } \\
\text { evaluación de las aulas virtuales son también poco utilizadas, solo en } \\
\text { casos puntuales el alumnado recibe información de sus calificaciones a } \\
\text { través del aula virtual. Sin embargo, el profesorado sí demanda la entrega } \\
\text { de muchas de las actividades que formarán parte de la evaluación a } \\
\text { través del aula virtual, lo que es valorado positivamente por el alumnado } \\
\text { por la facilidad que supone la entrega y el ahorro en impresión y } \\
\text { desplazamientos para su presentación. }\end{array}$ \\
\hline
\end{tabular}




\begin{tabular}{|c|c|}
\hline Dimensiones & Visiones del alumnado \\
\hline $\begin{array}{l}\text { Impacto en el } \\
\text { aprendizaje } \\
\text { y rol del } \\
\text { estudiante }\end{array}$ & $\begin{array}{l}\text { Una de las aportaciones más destacables de las aulas virtuales para el } \\
\text { alumnado es la disponibilidad del material de la asignatura, que ya no es } \\
\text { necesario imprimir o fotocopiar, y la entrega de actividades en formato } \\
\text { digital que pueden entregar a través del aula virtual sin necesidad de } \\
\text { desplazarse a la Facultad. } \\
\text { La disponibilidad de los materiales ha generado cierto grado de } \\
\text { absentismo, sin embargo, los estudiantes valoran el contacto cara a cara } \\
\text { con el profesorado. } \\
\text { Con el uso de las aulas virtuales, el profesorado sobrecarga de información } \\
\text { las aulas virtuales, que en ocasiones no se organizan ni identifican } \\
\text { adecuadamente, dificultándose la accesibilidad y generándose confusión } \\
\text { en el alumnado, que no sabe distinguir los materiales de estudio de los } \\
\text { materiales complementarios. }\end{array}$ \\
\hline $\begin{array}{l}\text { Opinión sobre } \\
\text { el aula virtual }\end{array}$ & $\begin{array}{l}\text { Los aspectos positivos recogidos en las entrevistas grupales son } \\
\text { la disponibilidad de los materiales didácticos de la asignatura, el } \\
\text { seguimiento y la entrega de actividades a través del aula virtual. } \\
\text { El alumnado indica que no todas las asignaturas utilizan aula virtual y que } \\
\text { en las asignaturas en las que se utiliza no se aplica de la misma forma y en } \\
\text { ocasiones el contenido se presenta de forma desorganizada, todo lo cual } \\
\text { genera confusión. } \\
\text { También se plantea la necesidad de ofrecer información específica para } \\
\text { el alumnado sobre los procedimientos de acceso y funcionamiento del } \\
\text { campus virtual a principio de curso. }\end{array}$ \\
\hline Expectativas & $\begin{array}{l}\text { El alumnado percibe un aumento del uso de las aulas virtuales. } \\
\text { Demanda necesidades de formación del profesorado para mejorar la } \\
\text { estructura y aprovechamiento del potencial de las aulas virtuales. } \\
\text { El alumnado habla de la tendencia a ausentarse de las clases cuando } \\
\text { dispone de los recursos en el aula virtual, ya que el profesorado no } \\
\text { controla la asistencia y las clases reproducen los aspectos contemplados } \\
\text { en el contenido. } \\
\text { Por otro lado, en las titulaciones de Grado en las que es necesario asistir } \\
\text { a clase el alumnado tiene mayores dificultades para compaginar trabajo } \\
\text { con estudios. Lo que hace pensar en la necesidad de configurar ofertas } \\
\text { formativas que se adapten a un alumnado con características diversas, } \\
\text { en este sentido las modalidades semipresenciales y en línea pueden dar } \\
\text { respuesta a estos problemas. }\end{array}$ \\
\hline
\end{tabular}

\section{Conclusión}

Las aulas virtuales, en los casos analizados, se conciben principalmente como espacios digitales de complementarios de la docencia presencial y aunque el alumnado valora positivamente su existencia (sobre todo para acceso a los materiales de estudio y como mecanismo de entrega de tareas y trabajos), demanda un mayor 
desarrollo en la dimensión de comunicación así como una mayor organización y calidad de los materiales de estudio.

En este estudio, las aulas virtuales de la docencia presencial funcionan más como un apéndice o apoyo ad hoc al modelo de enseñanza tradicional, que como revulsivo o catalizador de la innovación pedagógica de la docencia universitaria. En las mismas, sigue siendo predominante un modelo de enseñanza expositivo del conocimiento sin que se haya producido el salto hacia enfoques pedagógicos centrados en el estudiante que favorezcan procesos de aprendizaje autónomo, activos y sociales. Este es un conocimiento ya evidenciado en otros trabajos, que se ratifica en el caso de la universidad estudiada. Lo que nos lleva a inferir que estos fenómenos no son específicos de un contexto universitario particular (en nuestro caso, la ULL), sino que están generalizados en la mayor parte de instituciones presenciales de educación superior.

A pesar de la generalización de la incorporación a la docencia de las aulas virtuales entre gran parte del profesorado universitario, queda mucho camino por recorrer. En esta dirección el informe UNIVERSITIC 2016 sugiere que las universidades deben incidir en un mayor nivel de desarrollo (Gómez Arteaga, 2016) en favorecer prácticas generalizadas de calidad en la docencia virtual.

Por ello, hemos de concluir que es necesario desarrollar políticas institucionales desde la propia universidad para estimular no solo la creación y uso de estos entornos digitales, sino $-\mathrm{y}$ sobre todo- impulsar prácticas de metodologías didácticas y evaluativas que sean organizativa y pedagógicamente innovadoras. Esto implicará, como han señalado distintos informes (Pallof y Pratt, 2013; European Comission, 2014; Bates, 2015; Ossiannilsson, Camilleri, y Brown, 2015; Zawacki-Richter y otros 2014, 2016), un profundo rediseño de los contextos institucionales bajo los que enseñar. En este sentido, consideramos que la denominada enseñanza presencial debiera avanzar hacia modelos de b-learning, enseñanza mixta o semipresencial (Bartolomé, 2008; Vaughan, Cleveland-Innes, y Garrison, 2013; García Aretio, 2018), que constituye el punto intermedio entre una enseñanza a distancia virtual y una enseñanza totalmente presencial; así como también evolucionar hacia lo que Cabero (2013) denomina elearning 2.0.

También hemos de señalar que en el Informe Horizon 2017 (Becker y otros, 2017) se plantean algunas líneas de desarrollo en las plataformas LMS que suponen un salto cualitativo con relación a la actual generación de entornos formales de aulas virtuales. La incorporación de interfaces más visuales e interactivas, el diseño de entornos adaptativos y personalizables, la utilización de las analíticas del aprendizaje, entre otras, son elementos a incorporar en la próxima versión de aulas virtuales que favorezcan y permitan el desarrollo de metodologías emergentes e innovadoras para la docencia universitaria, tanto presencial como a distancia.

El estudio realizado tiene sus limitaciones, entre ellas, que solo ofrece la perspectiva del alumnado sobre la docencia virtual. En este sentido, será interesante como línea de investigación futura incorporar la perspectiva de los docentes sobre 
el diseño e implementación de la docencia virtual de cara a triangular las visiones de los distintos agentes educativos. Por otra parte, como acabamos de apuntar, la llegada de una nueva generación de plataformas de enseñanza en línea basadas en las interfaces multimedia, en analíticas de datos, así como en la inteligencia artificial generarán nuevos retos docentes, que deben ser explorados en futuros estudios. Finalmente, el contexto institucional tiene una poderosa influencia sobre las prácticas de docencia del profesorado, por lo que habremos de plantearnos cómo y de qué forma las políticas propias de las universidades presenciales pueden impulsar la generalización de modelos pedagógicos innovadores en los espacios virtuales de la educación superior de forma que se combinen y entremezclen las modalidades de enseñanza presencial con la formación a distancia digital de calidad educativa.

\section{REFERENCIAS BIBLIOGRÁFICAS}

Adams, S., Cummins, M., Davis, A., Freeman, A., Hall Giesinger, C., y Ananthanarayanan, V. (2017). NMC Horizon Report. 2017 Higher Education Edition. Austin, Texas: The New Media Consortium.

Adell, J., y Sales, A. (1999). El profesor online: elementos para la definición de un nuevo rol docente. Comunicación al III Congreso EDUTEC, Málaga.

Aguaded, I., y Cabero, J. (Coord.) (2013). Tecnologías y medios para la educación en la e-sociedad. Madrid: Alianza Editorial.

Aguaded, I., y Cabero, J. (Dir.) (2002). Educar en red. Internet como recurso para la Educación. Málaga: Aljibe.

Area, M., y Adell. J. (2009). E-Learning: Enseñar y aprender en espacios virtuales. En J. De Pablos (Coord.) Tecnología Educativa. La formación del profesorado en la era de Internet. (391-424). Málaga: Aliibe. Recuperado de https://www.researchgate.net/ publication/216393113 E-learning ensenar y aprender en espacios virtuales

Area, M., San Nicolás, M. B., y Fariña, E. (2008). Evaluación del Campus Virtual de la Universidad de La Laguna. Análisis de las aulas virtuales (Periodo 2005-
2007). San Cristóbal de La Laguna. Unidad para la Docencia Virtual.

Area, M., San Nicolás, M. B., y Fariña, E. (2010). Buenas prácticas de aulas virtuales en la docencia universitaria semipresencial. Teoría de la Educación. Educación y Cultura en la Sociedad de la Información, 11(1), 7-31.

Area, M., Sanabria, A., y González, M. (2008). Análisis de una experiencia de docencia universitaria semipresencial desde la perspectiva del alumnado. RIED. Revista iberoamericana de educación a distancia, 11(1), 231-254.

Barberá, E. (2008). Aprender e-learning. Barcelona: Paidós.

Barberá, E., Mauri, T., y Onrubia, J. (2008). Cómo valorar la calidad de la enseñanza basada en las TIC. Pautas e instrumentos de análisis. Barcelona: Graó.

Bartolome, A. (2008). Entornos de aprendizaje mixto en educación superior. RIED Revista Iberoamericana de Educación a Distancia, 11(1), 15-51. Recuperado de http://revistas.uned.es index.php/ried/article/view/955/874

Bates, A. (2015). Teaching in a Digital Age: Guidelines for Teaching and Learning. Victoria BC: BCcampus. Recuperado de: https://opentextbc.ca teachinginadigitalage 
Bates, A., y Sangrà, A. (2011). Managing Technology in Higher Education: Strategies for Transforming Teaching and Learning. San Francisco: John Wiley \& Sons.

Belmonte Nieto, M. (2002). Enseñar a Investigar. Orientaciones prácticas. Bilbao: Mensajero.

Cabero, C. (2013). La formación virtual en el entramado 2.0: el elearning 2.0. En J. Aguaded y J. Cabero (Coord.), Tecnologías y medios para la educación en la e-sociedad, 23-51. Madrid: Alianza Editorial.

Cabero, J. (2006). Bases pedagógicas del e-learning. RUSC. Revista de Universidad y Sociedad del Conocimiento, 3(1). Recuperado de http://www.redalyc.org/ html $/ 780 / 78030102 /$

Cabero, J., y Gisbert, M. (2005). La formación en Internet. Guía para el diseño de materiales didácticos. Sevilla: MAD.

Cabero, J., y Román, P. (2006). E-actividades. Sevilla: Eduforma,

Coll, C., y Monereo, C. (Ed.) (2008). Psicología de la educación virtual: aprender y enseñar con las tecnologías de la información y la comunicación. Madrid: Morata.

Duart, J. M., y Sangrá, A. (Comp.) (2000). Aprender en la virtualidad. Barcelona: Gedisa.

Fariña, E., González, C. S., y Area, M. (2013). ¿Qué uso hacen de las aulas virtuales los docentes universitarios? RED, Revista de Educación a Distancia, 35. Recuperado de http://www.um.es/ead/red/35/

García Aretio, L. (2018). Blended learning y la convergencia entre la educación presencial y a distancia. RIED Revista Iberoamericana de Educación a Distancia, 21(1), 9-22. Recuperado de http://revistas.uned.es/index.php/ried/ article/view/19683/16899

García Aretio, L. (Coord.), Ruiz Corbella, M., y Domínguez Figaredo, D. (2007). De la educación a distancia a la educación virtual. Barcelona: Ariel.

Gisbert Cervera, M. (2002). El nuevo rol del profesor en entornos tecnológicos. Acción Pedagógica, 11(1), 48-59.

Gómez Arteaga, J. (Dir.) (2016). UNIVERSITIC 2016. Análisis de las TIC en las Universidades Españolas. Madrid: $\quad$ Crue Universidades Españolas. Recuperado de http:/ www.crue.org/Documentos\%20 compartidos/Publicaciones/ Universitic/UNIVERSITIC\%202016 versi\%C3\%B3n\%20digital.pdf

Gorad, S., y Taylor, C. (2004). Combining Methods in Educational Research. Maidenhead: Open University Press.

Hoffman, S. J. (2010). Teaching the Humanities Online: A Practical Guide to the Virtual Classroom. Nueva York: Routledge.

Molnar, A. (Ed.), Miron, G., Gulosino, C., Shank, C., Davidson, C., Barbour, M. K., Huerta, L., Shafter, S. R., Rice, J. K., y Nitkin, D. (2017). Virtual Schools Report 2017. Colorado: National Education Policy Center. Recuperado de http:/ nepc.colorado.edu/publication/virtualschools-annual-2017

Ossiannilsson, E., Williams, K., Camilleri, A. F., y Brown, M. (2015): Quality models in online and open education around the globe: State of the art and recommendations. Oslo: International Council for Open and Distance Education. Recuperado de https:/ www.icde.org/assets/WHAT WE DO/ icdequalitymodels22.pdf

Palloff, R. M., y Pratt, K. (2013): Lessons from the virtual classroom: The realities of online teaching. San Francisco: JosseyBass

Píriz Durán, S. (Dir.) (2015). UNIVERSITIC 2015. Análisis de las TIC en las Universidades Españolas. Madrid: Crue Universidades Españolas. Recuperado de http://www.crue.org/Documentos\%20 
compartidos/Publicaciones/Universitic/ UNIVERSITIC 2015.pdf

Revuelta, F. I., y Pérez, L. (2009). Interactividad en los entornos de formación on-line. Barcelona: UOC.

Rodríguez, Ana P., Monteiro, A., y Moreira, J. A. (2013): Dimensões pedagógicas da sala de aula virtual: teoria e prática. Pedagogia no Ensino Superior, 26, 3-24 Recuperado de https://repositorioaberto. uab.pt/bitstream/10400.2/2744/3/ \begin{tabular}{lllllllllllll}
\hline $\mathrm{D}$ & $\mathrm{i} \mathrm{m}$ e & $\mathrm{n}$ & $\mathrm{s}$ & $\mathrm{o}$ & $\%$ & $\mathrm{C}$ & $\mathrm{C}$ & $\%$ & 8 & 3 & $\mathrm{e}$ & $\mathrm{s}$ \\
\hline \hline
\end{tabular} pedago\% C C \% 81 g i c a s cadernospedagogia\%20no\%20 ensino\%20superior\%281\%29.pdf

Salinas, J. (2004): Cambios metodológicos con las TIC. Estrategias didácticas $\mathrm{y}$ entornos virtuales de enseñanzaaprendizaje. Bordón, 56(3-4), 469-481.

Salinas, J., Darder A., y De Benito B. (2015). Las TIC en la enseñanza superior: e-learning, b-learning y m-learning. En J. Cabero y J. Barroso (Ed.), Nuevos retos en tecnología educativa (153-174). Madrid: Síntesis.

San Nicolás, M. B., Fariña Vargas, E., y Vera González, S. (2011). Desarrollo y evolución del Campus Virtual de la Universidad de La Laguna (2005-2010). $2^{O}$ Congreso Internacional Uso y Buenas Prácticas con TIC. Málaga: Facultad de Ciencias de la Educación. Universidad de Málaga.

Suárez, C., y Gros, B. (2013). Aprender en red. De la interacción a la colaboración. Barcelona: UOC.

Vassiliou, A., y McAlleese, M. (2014). High Level Group on the Modernisation of Higher Education. Report to the European Commission on New modes of learning and teaching in higher education. Comisión Europea, Luxemburgo. Recuperado de http:/ ec.europa.eu/dgs/education culture/ repositorv/education/librarv/reports/ modernisation-universities en.pdf

Zawacki-Richter, O., y Anderson, T. (Ed.) (2014). Online Distance Education: Towards a Research Agenda. Edmonton: AU Press, Athabasca University. Recuperado de http://www.aupress.ca/ books/120233/ebook/99Z ZawackiRichter Anderson 2014-Online Distance Education.pdf

Zawaki-Richer, O., y Naidu, S. (2016). Mapping research trends from 35 years of publications in Distance Education. Distance Education. 37(3), 245-269. Recuperado de http://www.tandfonline.com/doi/fu11/10.1080/01587919.2016.1185079?scrol=top\&needAccess =true

\section{PERFIL ACADÉMICO Y PROFESIONAL DE LOS AUTORES}

Manuel Area Moreira. Dr. en Pedagogía y Catedrático de Didáctica y Organización Escolar de la ULL. Es Director del grupo de investigación Laboratorio de Educación y Nuevas Tecnologías (EDULLAB), y de la Cátedra de Tecnología y Educación (TECNOEDU) de la Fundación Mapfre Guanarteme en la ULL. E-mail: manarea@ull.edu.es

María Belén San Nicolás Santos. Dra. en Pedagogía y Profesora del Departamento de Didáctica e Investigación Educativa de la ULL. Es miembro del grupo de investigación EDULLAB.

E-mail: bsannico@ull.edu.es 
Ana Luisa Sanabria Mesa. Dra. en Pedagogía y Profesora del Departamento de Didáctica e Investigación Educativa de la ULL Es miembro del grupo de investigación EDULLAB. Actualmente es Vicedecana de la Facultad de Educación en la ULL.

E-mail: asanabri@ull.edu.es

\section{DIRECCIÓN DE LOS AUTORES}

Facultad de Educación

Universidad de La Laguna

Avda. Avd. Trinidad s/n. 38204

San Cristóbal de La Laguna (España)

Fecha de recepción del artículo: 15/12/2017

Fecha de aceptación del artículo: 28/01/2018

\section{Como citar este artículo:}

Area, M., San Nicolás, B., y Sanabria, A. (2018). Las aulas virtuales en la docencia de una universidad presencial: la visión del alumnado. RIED. Revista Iberoamericana de Educación a Distancia, 21(2), pp. 179-198. doi: http://dx.doi. org/10.5944/ried.21.2.20666 


\section{ANEXO 1: Instrumento de análisis de las aulas virtuales}

\begin{tabular}{|c|c|}
\hline $\begin{array}{l}\text { Datos de } \\
\text { identificación }\end{array}$ & $\begin{array}{l}\text { Titulación } \\
\text { Rama de conocimiento } \\
\text { Curso } \\
\text { Número de alumnos y alumnas } \\
\text { Número de profesores }\end{array}$ \\
\hline $\begin{array}{l}\text { Objetos } y \\
\text { recursos de } \\
\text { información }\end{array}$ & $\begin{array}{l}\text { Objetos aprendizaje facilitados por el profesor: textos, presentaciones, } \\
\text { vídeos, material interactivo, otros,... } \\
\text { Uso de diferentes recursos propios del aula virtual. }\end{array}$ \\
\hline $\begin{array}{l}\text { Organización } \\
\text { didáctica }\end{array}$ & $\begin{array}{l}\text { Estructura del aula virtual (temas, semanal, social). } \\
\text { Guía docente de la asignatura. } \\
\text { Secuencia organizativa de las unidades didácticas (lecciones, temas, } \\
\text { proyectos, ...). } \\
\text { Calendario. } \\
\text { Coordinación docente (en caso de que sea un aula virtual compartida } \\
\text { entre varios docentes). }\end{array}$ \\
\hline \multirow[t]{2}{*}{$\begin{array}{l}\text { Modelo de } \\
\text { aprendizaje }\end{array}$} & $\begin{array}{l}\text { Modelo de aprendizaje predominante en función de las actividades } \\
\text { desarrolladas en el aula virtual. } \\
\text { - Aprendizaje por recepción. } \\
\text { - Aprendizaje por descubrimiento. } \\
\text { - Aprendizaje cooperativo. }\end{array}$ \\
\hline & Observaciones. \\
\hline $\begin{array}{l}\text { Tareas y } \\
\text { actividades }\end{array}$ & $\begin{array}{l}\text { Número de tareas y actividades. } \\
\text { Número de entregas. } \\
\text { Agrupamiento de estudiantes para cumplimentar actividades. } \\
\text { Tipo de actividades: búsqueda de información, análisis, reflexión, } \\
\text { comentario, ensayo, planificación y desarrollo de proyectos, debates, } \\
\text { resolución de ejercicios, cuestionarios de opinión, creación de glosarios, } \\
\text { trabajos en equipo, otros, ... } \\
\text { ¿En qué consisten las tareas y actividades planteadas en el aula virtual? }\end{array}$ \\
\hline $\begin{array}{l}\text { Funciones } \\
\text { de los } \\
\text { participantes }\end{array}$ & $\begin{array}{l}\text { Funciones desarrolladas por el profesor: transmitir información, } \\
\text { seguimiento, tutorización, evaluación, etc. } \\
\text { Funciones del estudiante: recepción información, desarrollo de proyectos } \\
\text { y tareas, cumplimentación de ejercicios y pruebas de evaluación, } \\
\text { participación en foros, etc. }\end{array}$ \\
\hline $\begin{array}{l}\text { Recursos de } \\
\text { comunicación }\end{array}$ & $\begin{array}{l}\text { Comunicación asíncrona: uso del foro de novedades, proposición y } \\
\text { uso de otros foros de aprendizaje para la interacción del alumnado y el } \\
\text { profesorado. } \\
\text { Número de foros de aprendizaje. } \\
\text { Media de mensajes en los foros. } \\
\text { Observaciones sobre la participación. } \\
\text { Comunicación síncrona: chat, videoconferencia, etc. } \\
\text { En caso afirmativo ¿̇qué recursos de comunicación síncrona se utilizan? }\end{array}$ \\
\hline
\end{tabular}




\begin{tabular}{|l|l|}
\hline $\begin{array}{l}\text { Recursos } \\
\text { externos al aula } \\
\text { virtual }\end{array}$ & $\begin{array}{l}\text { Uso de recursos externos: aplicaciones, servicios web para el desarrollo } \\
\text { de actividades de la asignatura. }\end{array}$ \\
\hline $\begin{array}{l}\text { Evaluación y } \\
\text { tutorización }\end{array}$ & $\begin{array}{l}\text { Objeto de la evaluación: del aprendizaje del alumnado, del aula virtual, } \\
\text { del desarrollo de la asignatura, del desempeño del profesor. } \\
\text { Criterios, estructura y uso del libro de calificaciones. } \\
\text { Procedimientos de evaluación del aprendizaje de los estudiantes. } \\
\text { Cantidad y frecuencia de la retroalimentación al alumnado de los } \\
\text { resultados de evaluación de las tareas. } \\
\text { Instrumentos de evaluación. }\end{array}$ \\
\hline $\begin{array}{l}\text { Aspectos } \\
\text { gráficos }\end{array}$ & $\begin{array}{l}\text { Diseño títulos, imágenes, enlaces, vídeos, materiales interactivos). } \\
\text { Tamaño de letra (pequeño, mediano, grande). } \\
\text { Uso de colores y contraste. } \\
\text { Observaciones. }\end{array}$ \\
\hline $\begin{array}{l}\text { Modelo } \\
\text { pedagógico }\end{array}$ & $\begin{array}{l}\text { Modelo pedagógico predominante en el aula virtual: } \\
\text { Modelo expositivo: aula como entorno de presentación y repositorio } \\
\text { de objetos digitales de transmisión de información } \\
\text { Modelo experiencial: aula como entorno para desarrollo de proyectos } \\
\text { y tareas de aprendizaje autónomo por estudiantes } \\
\text { Modelo interacción social: aula como espacio de comunicación e } \\
\text { interacción para el aprendizaje cooperativo entre estudiantes }\end{array}$ \\
\hline Observaciones & $\begin{array}{l}\text { Recoge categorías emergentes que no se hayan tenido en cuenta en otros } \\
\text { apartados, así como la visión general de la actividad desarrollada en el } \\
\text { aula virtual. }\end{array}$ \\
\hline
\end{tabular}




\section{ANEXO 2: Guion de la entrevista grupal}

\begin{tabular}{|c|c|}
\hline Uso de las TIC & $\begin{array}{l}\text { ¿Qué tareas realizas con el ordenador? } \\
\text { ¿Existen diferencias entre las actividades que realizan hombres y mujeres } \\
\text { con tecnología? ¿Cuáles? ¿Por qué? } \\
\text { La dotación tecnológica de las Facultades es cada vez menos utilizada ¿aa } \\
\text { qué crees que se debe? ¿Qué implicaciones tendrá para la universidad? } \\
\text { ¿Quedará en desuso? } \\
\text { Las TIC ¿son más utilizadas para el ocio o para las actividades } \\
\text { académicas? } \\
\text { ¿Cómo valorarías el grado de competencia digital del alumnado } \\
\text { universitario? }\end{array}$ \\
\hline $\begin{array}{l}\text { Experiencia } \\
\text { previa con } \\
\text { aulas virtuales }\end{array}$ & $\begin{array}{l}\text { ¿Han utilizado con anterioridad aulas virtuales? ¿Dónde? ¿Qué } \\
\text { actividades han realizado? }\end{array}$ \\
\hline $\begin{array}{l}\text { Características } \\
\text { y usos del aula } \\
\text { virtual }\end{array}$ & $\begin{array}{l}\text { ¿Con qué frecuencia sueles acceder al Campus Virtual? } \\
\text { ¿Crees que existen diferencias entre la dedicación de mujeres y hombres? } \\
\text { ¿Por qué? } \\
\text { ¿El tiempo de dedicación al aula virtual es igual al de las clases } \\
\text { presenciales, inferior o superior? ¿Por qué? } \\
\text { ¿Cuáles son los recursos del aula virtual que más utilizas? ¿Por qué? } \\
\text { ¿Cuáles son los recursos del aula virtual que menos utilizas? ¿Por qué? } \\
\text { ¿Qué actividades-tareas sueles hacer en el aula virtual? } \\
\text { ¿Favorecen el trabajo en equipo? ¿Cómo? ¿A través de qué recursos o } \\
\text { tareas? } \\
\text { ¿Se utilizan suficientemente los recursos de comunicación del aula } \\
\text { virtual? } \\
\text { ¿Por qué? }\end{array}$ \\
\hline $\begin{array}{l}\text { Impacto en el } \\
\text { aprendizaje }\end{array}$ & $\begin{array}{l}\text { ¿Qué cambios se han producido en los procesos de enseñanza y } \\
\text { aprendizaje con la utilización del aula virtual? } \\
\text { ¿En qué medida crees que el aula virtual ha mejorado tu aprendizaje? } \\
\text { ¿Cómo? ¿Cuáles crees que son las causas de estas mejoras? } \\
\text { ¿Qué recursos te resultan más útiles para tu aprendizaje? } \\
\text { ¿El alumnado se siente obligado a acceder a diario al aula virtual? ¿Por } \\
\text { qué? }\end{array}$ \\
\hline $\begin{array}{l}\text { Valoración } \\
\text { de las aulas } \\
\text { virtuales }\end{array}$ & $\begin{array}{l}\text { Aspectos positivos: ¿En qué medida las aulas virtuales te facilitan tu } \\
\text { trabajo como estudiante? } \\
\text { Aspectos negativos: ¿En qué medida te dificultan tu trabajo como } \\
\text { estudiante? }\end{array}$ \\
\hline Expectativas & $\begin{array}{l}\text { ¿En qué medida crees que se ha generalizado o tenderá a generalizarse el } \\
\text { uso de las aulas virtuales? ¿Por qué? } \\
\text { ¿Cursarías una titulación online? ¿Por qué? }\end{array}$ \\
\hline
\end{tabular}

